\title{
S GYNECOLOGICAL CANCER \\ Surgical approach of the left upper quadrant for ovarian cancer in 10 steps
}

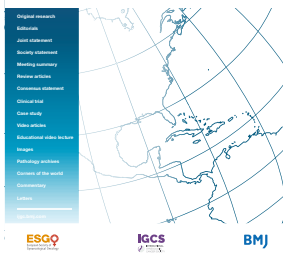

\section{Martina Aida Angeles (D) , ${ }^{1}$ Hélène Leray, ${ }^{1}$ Federico Migliorelli, ${ }^{2}$ Manon Daix,} Alejandra Martinez (ID) , ${ }^{1,3}$ Gwenael Ferron ${ }^{1,4}$

${ }^{1}$ Department of Surgical Oncology, Institut Universitaire du Cancer de ToulouseOncopole, Institut Claudius Regaud, Toulouse, Toulouse, Occitanie, France

${ }^{2}$ Department of Gynecology and Obstetrics, Paule de Viguier Hospital, Centre Hospitalier Universitaire de Toulouse, Toulouse, France ${ }^{3}$ INSERM CRCT Team 1 , Tumor Immunology and Immunotherapy, Toulouse, France

${ }^{4}$ INSERM CRCT Team 19, ONCOSARC-Oncogenesis of Sarcomas, Toulouse, France

\section{Correspondence to} Dr Gwenael Ferron, Surgical Oncology, Institut Claudius Regaud, 31059 Toulouse, Occitanie, France; ferron. gwenael@iuct-oncopole.fr

Accepted 20 September 2021 Published Online First 13 October 2021

\section{SUMMARY}

Complete cytoreductive surgery without residual tumor is one of the most important prognostic factors in advanced ovarian cancer patients. ${ }^{1}$ During cytoreductive procedures, it is not unusual to require multiple digestive and/or visceral resections, ${ }^{2}$ which undoubtedly increase surgical morbidity and can also decrease patients' quality of life. ${ }^{3}$ Therefore, it is essential to be as conservative as possible during cytoreductive procedures and to avoid, whenever possible, multivisceral resections without compromising the main goal of the surgery which is to achieve the absence of residual tumor. In this video article (Video 1) we show the surgical approach of the left upper quadrant in a patient with peritoneal carcinomatosis from ovarian origin who underwent an en-bloc left diaphragmatic peritonectomy, splenectomy, and infragastric omentectomy, among other procedures which are not included in the video. The surgery was carried out in a French Comprehensive Cancer Center by a senior oncological surgeon, following Sugarbaker principles. ${ }^{4}$ To perform the surgery, we used different energy

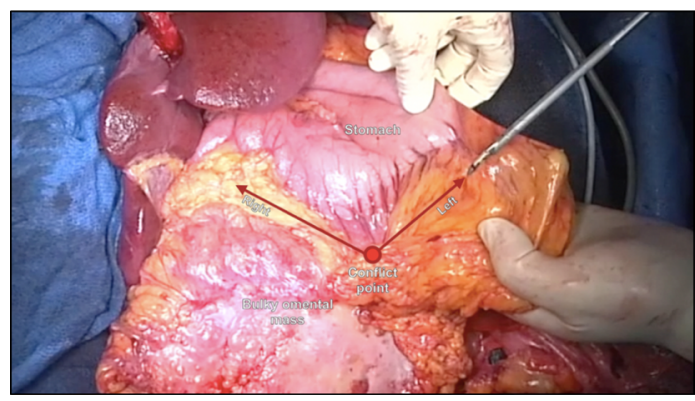

Figure 1 Infragastric omental detachment with gastroepiploic arcade preservation.

instruments, a multifunctional device which employs ultrasonic energy and advanced bipolar energy, a bipolar forceps, a monopolar tip, and a monopolar roller ball. We divided the surgical procedure in the 10 following steps:

Step 1: Surgical exposure

Step 2: Beginning of left diaphragmatic and paracolic gutter peritonectomy

Step 3: Mobilization of the splenic flexure of the colon

Step 4: Spleen mobilization

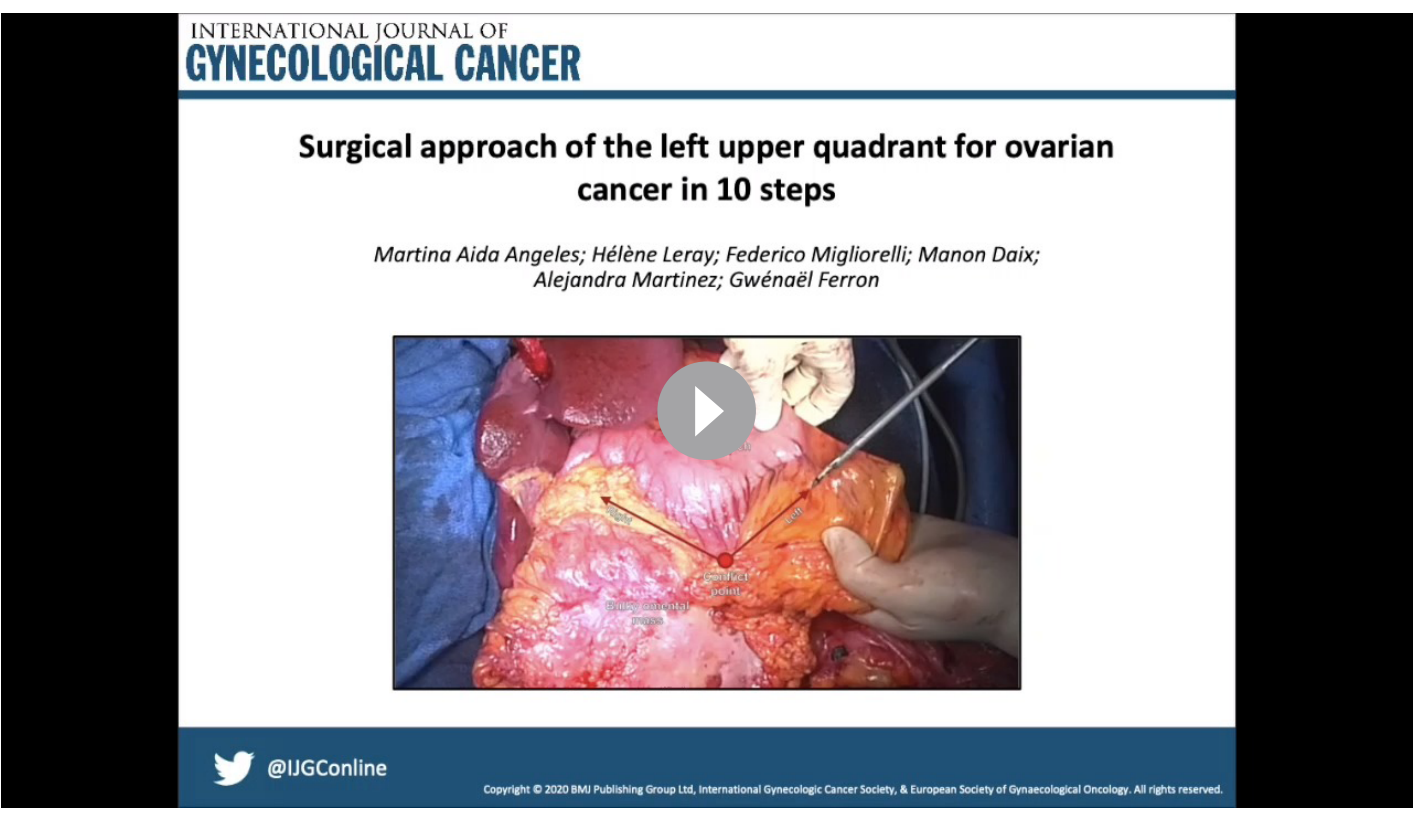

Video 1 Surgical approach of the left upper quadrant. 
Step 5: Complete left diaphragmatic peritonectomy

Step 6: Hepatic mobilization

Step 7: Infragastric omental detachment

Step 8: Splenic vascular pedicle ligature

Step 9: Coloepiploic detachment

Step 10: Chest tube insertion

To summarize, we propose an alternative surgical approach to spare a transverse colectomy in a patient who presented with an omental cake highly adherent to the transverse colon. It is crucial to reduce the surgical radicality of cytoreductive surgery in patients with advanced ovarian cancer, without hindering the oncological outcome.

Twitter Martina Aida Angeles @AngelesFite and Alejandra Martinez @Alejandra

Contributors MAA: Conceptualization, video editing, writing-original draft. $\mathrm{HL}$ : Conceptualization, video editing, writing-original draft. FM: Conceptualization, video editing, writing-original draft. MD: Conceptualization, video editing, writing-original draft. AM: Conceptualization, project administration, supervision, writing-review. GF: Conceptualization, project administration, surgery and video recording, supervision, writing-review.

Funding The authors have not declared a specific grant for this research from any funding agency in the public, commercial or not-for-profit sectors.
Competing interests GF reports personal fees from Olympus outside of the submitted work.

Patient consent for publication Consent obtained directly from patient(s)

Provenance and peer review Not commissioned; externally peer reviewed.

Data availability statement Data are available upon request.

\section{ORCID iDs}

Martina Aida Angeles http://orcid.org/0000-0003-4401-3084

Alejandra Martinez http://orcid.org/0000-0002-7633-3536

\section{REFERENCES}

1 Bristow RE, Tomacruz RS, Armstrong DK, et al. Survival effect of maximal cytoreductive surgery for advanced ovarian carcinoma during the platinum era: a meta-analysis. $J$ Clin Oncol 2002;20:1248-59.

2 Chi DS, Eisenhauer EL, Zivanovic O. Improved progression-free and overall survival in advanced ovarian cancer as a result of a change in surgical paradigm. Gynecol Oncol 2009;114:26-31.

3 Fagotti A, Ferrandina G, Vizzielli G, et al. Phase III randomised clinical trial comparing primary surgery versus neoadjuvant chemotherapy in advanced epithelial ovarian cancer with high tumour load (SCORPION trial): final analysis of peri-operative outcome. Eur J Cancer 2016;59:22-33.

4 Sugarbaker PH. Peritonectomy procedures. Ann Surg 1995;221:29-42. 